\title{
Prevalência de Hepatite B em Parturientes e Perfil Sorologico Perinatal
}

\author{
Prevalence of Hepatitis B in Parturients and Perinatal Serologic Profile \\ Luiz Cláudio Arraes ${ }^{1,2}$, Aletheia Soares Sampaio ${ }^{3}$, Silvana Barreto ${ }^{3}$, \\ Maria do Socorro Agra Guilherme ${ }^{2}$, Felipe Lorenzato ${ }^{2,4}$
}

\section{RESUMO}

\begin{abstract}
Objetivos: avaliar a prevalência do antigeno de superficie do virus $B$ da hepatite (HBsAg) em parturientes internadas no Instituto Materno-Infantil de Pernambuco (IMIP), Recife-PE, e o perfil sorológico das positivas.

Métodos: este é estudo de secção de corte transversal prospectivo no qual foram utilizados os sistemas VIDAS e VIDAS HBs, respectivamente, para detecção e confirmação do HBsAg. As participantes foram selecionadas de forma aleatória. Nas pacientes $\mathrm{HBs} \mathrm{Ag}^{+}$, os outros marcadores sorológicos foram testados com o sistema automatizado AxSYM. Os recém-nascidos de mães $H B s A g+$ foram vacinados ao nascer com a vacina Engerix $B$.

Resultados: de um total de 1584, encontramos 9 (0,6\%) gestantes HBsAg positivas. Nenhuma delas apresentava anti-HBc IgM, sendo assim casos prevalentes. Em 1/9 (11,1\%) das gestantes havia presença do HBeAg isolado e em 4/9 (44,4\%), deste antígeno e seu anticorpo correspondente, revelando os diferentes graus de risco de transmissão vertical da infecção. Com a exceção de dois recém-nascidos de um parto gemelar (um de baixo peso), todos apresentaram soroconversão para o anti-HBs com três doses da vacina. Os gemelares prematuros só mostraram soroconversão após a quarta dose da vacina.

Conclusões: a prevalência da hepatite $B$ em parturientes no IMIP é relativamente baixa $e$ todas as pacientes detectadas então tinham a forma crônica da infecção.
\end{abstract}

PALAVRAS-CHAVE: Hepatite B. Transmissão vertical. Rastreamento, hepatite. Vacinação, hepatite. Infecções na gravidez.

\section{Introdução}

A hepatite B é doença infecciosa, causada por um virus hepatotrópico, DNA, pertencente à familia Hepadnavíridae, de estrutura complexa. Pode apresentar-se sob diversas formas clinicas, sendo de maior preocupação as formas crônicas, responsáveis pela progressão para cirrose e carcinoma hepatocelular. Segundo a Organização

Departamentos de Imunologia ${ }^{1}$ e Obstetrícia ${ }^{4}$ e Mestrado em Saúde Materno-Infantil ${ }^{2}$ do Instituto Materno-Infantil de Pernambuco e Laboratório Municipal de Saúde Pública/ Mestrado em Medicina Tropical da $\mathrm{UFPE}^{3}$, Recife-PE.

Correspondência:

Luiz Cláudio Arraes

Departamento de Imunologia, Instituto Materno-Infantil de Pernambuco (IMIP)

Rua dos Coelhos, 300 - Boa Vista

50070-50 - Recife - PE

Fone: (81) 3413-2169 - Fax: (81) 3413-2180

e-mail: lularraes@hotmail.com
Mundial de Saúde (OMS), existem no mundo mais de 350 milhões de portadores crônicos do vírus $B$ da hepatite (VHB) e ocorrem mais de um milhão de mortes por ano causadas pela progressão da doença para cirrose e carcinoma hepatocelular ${ }^{1}$.

A prevalência de hepatite $B$ em gestantes varia de acordo com a endemicidade da infecção na região geográfica e população estudada ${ }^{2-4}$. Particularmente no Brasil, há poucos estudos de rastreamento de hepatite B em gestantes, já que os marcadores sorológicos para hepatite B não fazem parte dos exames de rotina do pré-natal em serviços públicos.

As vias de transmissão do VHB incluem contato sexual, seja hetero ou homossexual, caracterizando-a como doença sexualmente transmissivel, além de possivel contágio por contato com sangue ou hemoderivados infectados ${ }^{5-7}$. Merece importante destaque a transmissão vertical que, em termos mundiais, representa a principal via de disseminação do 
VHB nas regiões de altas prevalências ${ }^{8}$. Além disso, os neonatos, quando infectados, têm alto risco de desenvolver formas crônicas ( $90 \%$ dos casos), devido à imaturidade do seu sistema imunológico. A transmissão vertical ocorre predominantemente durante o parto, por meio de contato com sangue, líquido amniótico ou secreções maternas, sendo rara a transmissão via transplacentária, leite materno ou após o nascimento9.

Os riscos de transmissão vertical aumentam quando se tem alta carga viral materna, altos títulos de marcadores como o antígeno de superficie (HBsAg) e a presença do antígeno "e" (HBeAg), marcador de replicação viral. Além disso, o risco de infecção do concepto após uma hepatite B aguda, autolimitada, ocorrida na gravidez, depende do periodo gestacional em que a infecção ocorreu. É maior o risco se a mãe se infectar no terceiro trimestre. Neste caso, 80 a $90 \%$ dos neonatos serão HBsAg positivos, em comparação com $10 \%$ de positivos se ocorrer no primeiro trimestre ${ }^{10,11}$. Esta cronificação precoce leva a risco de aproximadamente $25 \%$ de evolução para cirrose ou hepatocarcinoma.

Do ponto de vista clínico, a associação de hepatite viral aguda e gravidez é pouco freqüente, porém, esta infecção é a causa mais comum de icterícia na gestação ${ }^{12}$. Na maioria dos casos, a hepatite $\mathrm{B}$ apresenta-se como assintomática ou com sinais e sintomas inespecíficos, como anorexia, náuseas, vômitos, inapetência e dores abdominais, dentre outros. A ictericia raramente está presente (20\% dos casos), o que dificulta bastante o diagnóstico, já que os referidos sintomas confundem-se com distúrbios fisiológicos comuns da própria gravidez. Até mesmo os sinais de doença hepática crônica, incluindo eritema palmar, esplenomegalia e telangiectasias aracneiformes, também são comuns durante o estado de hiperestrogenia fisiológica da própria gravidez. Por conta disso, muitas gestantes com VHB assintomáticas são identificadas de forma indireta, a partir de um achado de aminotransferases elevadas. Estas, por sua vez, nem sempre estarão alteradas, principalmente nos casos de mães portadoras crônicas assintomáticas, que são a maioria das grávidas que têm hepatite $B$. Isso porque as pacientes com doença hepática prévia avançada, com cirrose, têm menor fertilidade ${ }^{13}$.

Portanto, de maneira geral, o diagnóstico definitivo da hepatite $B$ só é possivel por meio da detecção dos marcadores sorológicos. Porém, no Brasil, conforme já mencionado, tal investigação ainda não faz parte do pré-natal de rotina nos serviços públicos, embora normatização recente indique o uso associado da imunoglobulina com a vaci- na em neonatos de mães HBsAg positivas. Nos Estados Unidos da América, o Centro de Controle de Doenças (CDC) e o Colégio Americano de Ginecologistas e Obstetras (ACOG) recomendam o rastreamento sorológico para hepatite $\mathrm{B}$ de todas as gestantes, independente dos fatores de risco. Esta medida permite aos recém-nascidos de mães positivas receberem imunoglobulina anti-hepatite B associada à vacina, o que reduz significativamente a transmissão vertical. Se tal procedimento for realizado nas primeiras doze horas de nascimento, reduz-se em mais de $90 \%$ a transmissão vertical ${ }^{14}$. Além disto, o rastreamento pré-natal de gestantes permite realizar imunização ativa (vacina) ou passiva (imunoglobulina) em mulheres suscetiveis, já que a vacina não é contra-indicada na gravidez.

O objetivo principal deste trabalho foi determinar a prevalência do antígeno HBsAg em parturientes. Também se objetivou estudar, entre as parturientes HBsAg positivas, a evolução do perfil sorológico para o anti-HBc (frações IgG e IgM), $\mathrm{HBeAg}$, anti-HBe e anti-HBs em dois momentos após o parto, com intervalo de seis meses, e determinar o perfil sorológico pós-vacinal (após a terceira dose da vacina Engerix B) dos lactentes de mães HBsAg positivas.

\section{Pacientes e Métodos}

Este trabalho foi um estudo descritivo, de corte transversal, realizado no período de junho a dezembro de 2001. O protocolo do estudo foi revisado e aprovado pela Comissão de Ética em Pesquisa local antes que a primeira participante tivesse sido incluída. A população em estudo foi constituída por 1584 parturientes internadas no Centro de Atenção à Mulher (CAM) do Instituto Materno-Infantil de Pernambuco (IMIP), localizado em Recife (Pernambuco). As pacientes, selecionadas de forma aleatória, foram informadas e concordaram em participar do estudo, tendo sido obtida autorização por escrito de cada uma delas, pela assinatura de termo de consentimento livre e esclarecido.

Os dados foram coletados utilizando formulários padronizados, pré-codificados para entrada de dados no computador. Após preenchidos os formulários, estes foram digitados em banco de dados específico criado no programa Epi-Info versão 6.04 e no SPSS 10.

As amostras das 1584 pacientes foram colhidas retirando-se, por punção venosa, $10 \mathrm{~mL}$ de sangue de cada paciente com seringa descartável e colocada em tubos estéreis apropriados. A 
detecção de antígeno HBsAg foi realizada no setor de imunologia do IMIP, por meio de aparelhagem computadorizada, denominada sistema VIDAS, a qual oferece resultado qualitativo tanto no soro como no plasma humano pela técnica ELFA/ELISA (Enzyme Linked Fluorescent Assay/Ensaio imunenzimático fluorescente). Nos casos de positividade, os testes foram repetidos e, após centrifugação da amostra, confirmados por teste confirmatório (VIDAS HBs).

O perfil sorológico materno foi realizado em dois momentos: em fevereiro de 2002 e nova coleta em setembro de 2002, só para as mães com sorologia positiva na primeira avaliação. As mães que tiveram o resultado positivo para o HBsAg e seus recém-nascidos eram submetidos à investigação sorológica de outros marcadores virais de hepatite B: o anti-HBc IgM, anti-HBc total, HBeAg, anti-HBe e anti-HBs. Tal investigação foi realizada no Laboratório Municipal de Saúde Pública do Recife, utilizando-se o sistema automatizado AxSYM para detecção de marcadores sorológicos da hepatite B.

Os recém-nascidos das mães HBsAg positivas foram analisados a partir da coleta de $2 \mathrm{~mL}$ de sangue obtida por punção venosa e depois imunizados com três doses da vacina Engerix B. A vacina foi administrada em três doses de $0,5 \mathrm{~mL}$, no músculo lateral da coxa, com intervalos de um mês entre a primeira e a segunda e seis meses entre a segunda e a terceira dose, conforme é feito no calendário vacinal de rotina. Tais recém-nascidos foram submetidos ao acompanhamento quantitativo da resposta imunológica à vacina, pela dosagem do anti-HBs, após um mês da terceira dose da vacina.

Os dados foram analisados de forma sumária e descritos em tabelas de distribuição de freqüências.

\section{Resultados}

Entre junho a setembro de 2001, 1584 gestantes foram avaliadas na hora do parto para o estudo de portadoras do HBV por meio do rastreamento do antígeno de superficie da hepatite $B$ no IMIP. A prevalência encontrada de mães $\mathrm{HBsAg+}$ revelou-se baixa, sendo igual a 0,6\% (9/1584) e com intervalo de confiança a $95 \%$ de 0,2 a $0,9 \%$.

O perfil sorológico das mães soropositivas (HBsAg+) em fevereiro de 2002 (Tabela 1) revelou a presença do anti-HBc total em todas as gestantes. Quando se considerou a presença isolada do HBeAg e do anti-HBe, os percentuais encontrados foram $11,1 \%(1 / 9)$ e $44,4 \%$ (4/9), respectivamente. Em 44,4\% (4/9) dos casos havia concomitantemente a presença de HBeAg e anti-HBe. Em nenhuma delas foi detectado o anti-HBc IgM nem o anti-HBs (Tabela 1).

Tabela 1 - Resultados do perfil sorológico de marcadores do vírus da hepatite B (HBV) em mães soropositivas para o antígeno HBsAg no IMIP.

\begin{tabular}{|c|c|c|c|c|c|c|c|}
\hline Paciente & HBsAg & Anti-HBc IgM & Anti-HBc & HBeAg Fevereiro & HBeAg Setembro & Anti-HBe & Anti-HBs \\
\hline A & $\mathrm{R}$ & NR & $\mathrm{R}$ & $N R$ & NR & $\mathrm{R}$ & NR \\
\hline B & $\mathrm{R}$ & $N R$ & $\mathrm{R}$ & $\mathrm{R}$ & $\mathrm{R}$ & $N R$ & $N R$ \\
\hline C & $\mathrm{R}$ & $N R$ & $\mathrm{R}$ & $\mathrm{R}$ & $\mathrm{R}$ & $\mathrm{R}$ & NR \\
\hline D & $\mathrm{R}$ & NR & $\mathrm{R}$ & $\mathrm{R}$ & $\mathrm{R}$ & $\mathrm{R}$ & NR \\
\hline E & $\mathrm{R}$ & $N R$ & $\mathrm{R}$ & $N R$ & NR & $\mathrm{R}$ & NR \\
\hline $\mathrm{F}$ & $R$ & $N R$ & $\mathrm{R}$ & $\mathrm{R}$ & $\mathrm{R}$ & $\mathrm{R}$ & NR \\
\hline G & $\mathrm{R}$ & $N R$ & $\mathrm{R}$ & $N R$ & $N R$ & $\mathrm{R}$ & NR \\
\hline $\mathrm{H}$ & $\mathrm{R}$ & NR & $\mathrm{R}$ & $\mathrm{R}$ & $\mathrm{R}$ & $\mathrm{R}$ & NR \\
\hline I & $\mathrm{R}$ & NR & $\mathrm{R}$ & $N R$ & NR & $\mathrm{R}$ & NR \\
\hline
\end{tabular}

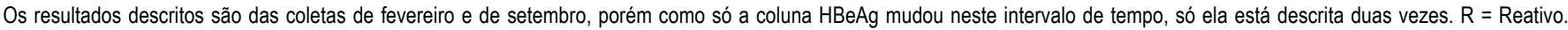
NR = Não reativo.

Em setembro de 2002, realizou-se nova coleta sanguínea nas mães soropositivas e outro perfil sorológico materno que confirmou em todas a presença do anti-HBc total. Em 88,9\% (8/9) detectou-se a presença do anti-HBe e em apenas $11,1 \%(1 / 9)$ a presença do HBeAg sem anti-HBe. Em nenhuma delas foi encontrado o anti-HBc IgM, nem o anti-HBs (Tabela 1).
Os lactentes das mães com soropositividade para o HBsAg foram acompanhados e avaliados após a terceira dose da vacina de DNA recombinante do laboratório SmithKline em setembro de 2002. O perfil sorológico pós-vacinal desses lactentes encontra-se ilustrado na Tabela 2. Do total de 10 lactentes (uma gestação foi gemelar) das 9 mães HBsAg+, 8 (80\%) obtiveram soroproteção pós- 
vacinal, porém dois lactentes $(20 \%)$, exatamente os dois gêmeos prematuros, não apresentaram títulos detectáveis de anticorpos anti-HBs considerados soroprotetores e por este motivo foram revacinados ( $4^{\mathrm{a}}$ dose). Nenhum dos lactentes apresentou anticorpos anti-HBc IgM. Dos 8 lactentes que apresentaram anticorpos anti-HBs, 62,5\% $(5 / 8)$ também foram reagentes para os anticorpos anti-HBc total. Destes, 37,5\% (3/8) apresentaram soropositividade para o anti-HBe, ou seja, eram positivos para anti-HBs, anti-HBc total e anti-HBe simultaneamente. Apenas dois lactentes (20\%) apresentaram anticorpos anti-HBs isoladamente. Nos dois lactentes que não apresentaram anticorpos anti-HBs (os gêmeos), foi detectada a presença de anticorpos anti-HBc total e anti-HBe. Os gemelares foram revacinados ( $4^{a}$ dose) e apresentaram niveis de anti-HBs protetores apenas com esta dose suplementar. Nenhum lactente apresentou antígeno HBsAg e $\mathrm{HBeAg}$ reativos.

Tabela 2 - Resultados do perfil sorológico de marcadores do vírus da hepatite B (HBV) nos lactentes de mães soropositivas para o antígeno HBsAg no IMIP.

\begin{tabular}{|c|c|c|c|c|c|c|}
\hline Lactente & HBsAg & Anti-HBc IgM & Anti-HBc & $\mathrm{HBeAg}$ & Anti-HBe & Anti-HBs \\
\hline$A$ & $\mathrm{NR}^{*}$ & $N R$ & $\mathrm{R}$ & NR & $\mathrm{R}$ & NR \\
\hline B & $\mathrm{NR}^{*}$ & $N R$ & $\mathrm{R}$ & NR & $\mathrm{R}$ & $N R$ \\
\hline C & $N R$ & NR & $N R$ & $N R$ & $N R$ & $\mathrm{R}$ \\
\hline D & NR & NR & $\mathrm{R}$ & $N R$ & $\mathrm{R}$ & $\mathrm{R}$ \\
\hline $\mathrm{E}$ & NR & $N R$ & $\mathrm{R}$ & NR & $\mathrm{R}$ & $\mathrm{R}$ \\
\hline $\mathrm{F}$ & $N R$ & $N R$ & $\mathrm{R}$ & $N R$ & $N R$ & $\mathrm{R}$ \\
\hline G & $N R$ & $N R$ & NR & NR & NR & $\mathrm{R}$ \\
\hline $\mathrm{H}$ & NR & NR & $\mathrm{R}$ & NR & NR & $\mathrm{R}$ \\
\hline 1 & NR & NR & NR & NR & NR & $\mathrm{R}$ \\
\hline J & NR & $N R$ & $\mathrm{R}$ & NR & $\mathrm{R}$ & $\mathrm{R}$ \\
\hline
\end{tabular}

$R=$ Reativo. $N R=$ Não reativo. ${ }^{*} R N$ gemelar (G1 e G2) respectivamente.

\section{Discussão}

A baixa prevalência de mães com resultado positivo para o antígeno de superficie contra a hepatite $B$ na instituição de referência estudada $(0,6 \%)$ anima as autoridades sanitárias e levanta questionamentos quanto à relação custo-eficácia e quanto à auto-sustentabilidade de programa de vacinação em massa para população local. Porém, apesar de os resultados terem sido compativeis com os de outros estudos realizados em São Paulo, como o de Sabino et al. ${ }^{15}$, que encontraram 0,4\% de prevalência; no Rio de Janeiro, como o de Oliveira et al. ${ }^{16}$, que encontraram $0,6 \%$, e em Goiânia, como o de Cardoso et al. ${ }^{17}$, que encontraram 0,5\%, novos estudos de prevalência do HBsAg na população obstétrica de Recife seriam necessários para constatar se essa baixa prevalência reflete a realidade em escala mais ampla. Dados discordantes do nosso e desses outros citados, provavelmente devido às diferentes populações estudas, tamanhos amostrais e às diferentes eficácias de métodos laboratoriais utilizados, foram encontrados no estado do Amazonas, onde a prevalência é conhecidamente alta para o virus $\mathrm{B}$, como descre- vem Hardler et al. ${ }^{18}$, que encontraram uma prevalência de $3,2 \%$ de gestantes HBsAg positivas dentre um total de 1460 gestantes pesquisadas. Da Silva ${ }^{19}$ encontrou $1,7 \%$ de gestantes soropositivas para o HBsAg em Salvador. A importância de mais estudos sobre o assunto, com método de detecção padrão e maiores amostras, fica patente devido à discordância dos dados de nosso estudo com outros, como o de Machado-Júnior ${ }^{20}$, que encontrou em outra maternidade do Recife prevalência de $3,2 \%$ (14/450), números que se assemelham mais aos da região norte do Brasil. O curto espaço de tempo entre um estudo e outro nos parece insuficiente para explicar eventual impacto direto ou indireto da vacinação contra a hepatite B em amplas faixas etárias da população.

As mães soropositivas para o HBsAg foram submetidas à pesquisa sorológica dos outros marcadores virais da hepatite B em fevereiro de 2002 e setembro de 2002. Esta análise revelou que todas as gestantes $\mathrm{HbsAg+}$ incluídas no presente estudo eram portadoras crônicas da doença, visto que nenhuma era sintomática nem positiva para anti-HBc IgM. Baseado no perfil sorológico de fevereiro de 2002 (Tabela 1), o risco de infecção neonatal estava aumentado na maioria dos casos 
$(55,6 \%)$, pois estas gestantes tiveram sorologia positiva tanto para o $\mathrm{HBsAg}$ como o $\mathrm{HBeAg}$, o que confere alta infectividade. No entanto, das cinco pacientes positivas para $\mathrm{HBsAg}$ e $\mathrm{HBeAg}$, simultaneamente, só uma não resultou positiva para o anticorpo anti-HBe, o que conferia à mesma grande risco de transmissão da hepatite para seu bebê (cerca de 90\%). Já os recém-nascidos das outras quatro mães, que além da concomitância dos dois antígenos eram positivas para o anticorpo antiHBe, o qual oferece certa proteção contra a transmissão, tiveram risco ainda aumentado, mas bem menor ( 5 a $25 \%$ ) do que se não tivessem esta defesa imunológica. É importante ressaltar que os recém-nascidos que adquirem hepatite $B$ não respondem tão bem quanto os adultos, devido à imaturidade imunológica, e 90\% deles evoluem para o estado de portadores crônicos, assim se tornando capazes de infectar outras pessoas e, mais importante, eles têm $25 \%$ de chance de evoluir para cirrose ou carcinoma hepatocelular ${ }^{21}$. Na reavaliação do perfil sorológico materno 7 meses após o diagnóstico, a paciente $\mathrm{B}$ foi a única que continuou com potencial infectante e com a mesma magnitude inicial, portanto a mesma necessitará de seguimento criterioso.

Quanto ao perfil sorológico pós-vacinal, observamos o desenvolvimento de imunidade em $80 \%$ dos lactentes estudados, filhos de mães soropositivas para o HBsAg. Apenas um quinto destes lactentes não soroconverteram de imediato e foram exatamente os nascidos de parto gemelar e pré-termo. A prematuridade e o baixo peso ao nascer provavelmente são os principais fatores que influenciaram negativamente na soroconversão pós-vacinal ${ }^{22-25}$. Entretanto, tais recém-nascidos, supostamente com imunidade inferior à dos demais, soroconverteram mais tarde, após a quarta dose de vacina, demonstrando terem sido válidas as suas imunizações, tanto quanto à profilaxia pósexposição quanto à prevenção diante de possiveis exposições futuras.

A implementação do uso de imunoglobulina contra hepatite B associada à vacina aplicada aos recém-nascidos de mães soropositivas, conforme recomenda o Ministério da Saúde, requer rastreamento sorológico pré-natal universal do HBsAg materno para identificar os filhos de mães positivas e vaciná-los. Isso implica altos custos e, devido à baixa endemicidade, fica o questionamento se tal medida é custo-efetiva.

A maioria das gestantes $\mathrm{HBsAg}+$ neste estudo $(55,6 \%)$, mesmo sendo portadoras de hepatite B crônica, ofereciam um alto risco de transmissão da infecção para seus filhos, pois apresentavam simultaneamente os antígenos HBsAg e HBeAg. Uma pa- ciente permanceu com aproximadamente $90 \%$ de chance de transmissibilidade da doença mesmo 7 meses após o parto.

Após a terceira dose da vacina, houve desenvolvimento de imunidade em $80 \%$ dos lactentes estudados, filhos de mães soropositivas para o HBsAg. Os $20 \%$ restantes, apesar da prematuridade e baixo peso ao nascer, também soroconverteram, porém só após a aplicação da quarta dose da vacina.

Logo, a eficácia da vacina de DNA recombinante contra a hepatite $B$, mesmo sem a administração de imunoglobulina simultânea, na prevenção da transmissão vertical, foi demonstrada neste trabalho. Este dado pode ser útil como base científica para realização de futuros estudos. Conseqüentemente, a validação de tal estratégia, caso novos estudos revelem resultados semelhantes, além da constatação de baixa prevalência regional de HBsAg em gestantes, poderia permitir a adoção do uso rotineiro de vacinação sem imunoglobulina associada, o que implicaria redução importante nos custos.

\section{ABSTRACT}

Purpose: to estimate the prevalence of the hepatitis B surface antigen (HBsAg) in parturients admitted to the "Instituto Materno-Infantil de Pernambuco (IMIP)', Recife-PE, and to determine the serologic profile of the positive ones.

Methods: this is a prospective cross-sectional study where the VIDAS and VIDAS HBs systems were used for detection and confirmation of HBsAg, respectively. The parturients were randomly selected. In $\mathrm{HBs} \mathrm{Ag}+$ patients, the other serologic markers were tested by the use of the AxSYM automated system. The newborn babies of $\mathrm{HBs} \mathrm{Ag}+$ mothers were vaccinated with the Engerix $B$ vaccine.

Results: among 1584 parturients, there were $9(0.6 \%) \mathrm{HBs} \mathrm{Ag}$ positive. None of them had anti-HBc IgM, thus they were all prevalent cases. In 1/9 (11.1\%) of the HBsAg+ mothers, HBeAg was isolated and in 4/9 (44.4\%), this antigen circulated along with its antibody, hence the importance of establishing the different magnitudes of risk of vertical transmission. Except for two newborn babies from a twin pregnancy (one with low birth weight), all presented seroconversion to anti-HBs with 3 doses of the vaccine. The premature twin babies showed seroconvertion only after the fourth dose of the vaccine.

Conclusions: the prevalence of hepatitis $B$ among parturients at IMIP is relatively low and all patients diagnosed had the chronic form of the infection.

KEYWORDS: Hepatitis B. Vertical transmission. Screening, hepatitis. Vaccination, hepatitis. Infections in pregnancy. 


\section{Referências}

1. Sylvan S. WHO spearheads global initiative to eradicate hepatitis B. Lakartidningen 2000; 97:3738-40.

2. Euler GL, Wooten KG, Baughman AL, Williams WW. Hepatitis B surface antigen prevalence among pregnant women in urban areas: implications for testing, reporting, and preventing perinatal transmission. Pediatrics 2003; 111:1192-7.

3. Gotstein MG, Aide PM, Coleman PF, Sanborn MR. Rate of hepatitis $B$ virus infection in pregnant women determined by a monoclonal hepatitis B surface antigen immunoassay. J Clin Microbiol 2002; 40:3493-6.

4. Lin HH, Kao JH, Chang TC, Hsu HY, Chen DS. Secular trend of age-specific prevalence of hepatitis $\mathrm{B}$ surface and e antigenemia in pregnant women in Taiwan. J Med Virol 2003; 69:466-70.

5. van Hattum J, Boland GJ, Jansen KG, et al. Transmission profile of hepatitis $B$ virus infection in the Batam region, Indonesia. Evidence for a predominantly horizontal transmission profile. Adv Exp Med Biol 2003; 531:177-83.

6. Savas MC, Guney C, Kadayifci A, et al. High prevalence of transfusion-transmitted virus infection in patients with chronic liver diseases in an endemic area of hepatitis B and C virus. Med Princ Pract 2003; 12:176-9.

7. Russi JC, Serra M, Vinoles J, et al. Sexual transmission of hepatitis B virus, hepatitis $\mathrm{C}$ virus, and human immunodeficiency virus type 1 infections among male transvestite comercial sex workers in Montevideo, Uruguay. Am J Trop Med Hyg 2003; 68:716-20.

8. Darani M, Gerber M. Hepatitis B antigen in vaginal secretions. Lancet 1974; 2:1008.

9. Samuels P, Cohen AW. Pregnancies complicated by liver disease and liver dysfunction. Obstet Gynecol Clin North Am 1992; 19:745-63.

10. Sweet RL. Hepatitis B infection in pregnancy. Obstet Gynecol Rep 1990; 2:128.

11.Arevalo JA, Washington AE. Cost-effectiveness of prenatal screening and immunization for hepatitis B virus. JAMA 1988; 259:365-9.

12.Arevalo JA. Hepatitis B in pregnancy. West J Med 1988; 150:668-74.

13.Lee WM. Pregnancy in patients with chronic liver disease. Gastroenterol Clin North Am 1992; $21: 889$ 903.
14.Stevens CE, Taylor PE, Tong MJ, et al. Yeastrecombinant hepatitis $B$ vaccine. Efficacy with hepatitis B immune globulin in prevention of perinatal hepatitis $B$ virus transmission. JAMA 1987; 257:2612-6.

15.Sabino EC, Guerra EM, Oba IT, Spina AM, Vaz AJ. The incidence of hepatitis B markers in pregnant women at their first consultation in metropolitanarea health centers, São Paulo, Brazil. Rev Inst Med Trop São Paulo 1992; 34:535-41.

16. Oliveira ND, Kopelman BI, Mundin HC, Campos TMA. Prevalência de gestantes portadoras do vírus da hepatite B (VHB) e transmissão perinatal. J Pediatr (RJ) 1993; 69:53-60.

17. Cardoso DDP, Faria EL, Azevedo MSP, et al. Soroepidemiologia para o virus da hepatite B (VHB) em gestantes/parturientes e sua transmissão para recém-nascidos em Goiânia - GO. Rev Soc Bras Med Trop 1996; 29:349-53.

18. Hardler S, Fay OH, Pinheiro FP, Maynard JE. La hepatitis en las Americas: informe del grupo colaborador de la OPS. Bol Oficina Sanit Panam 1987; 103:185-209.

19.Da Silva LC. Management of viral hepatitis. Rev Paul Med 1984; 102:227-8.

20.Machado-Júnior B. Soropositividade para hepatite a vírus B em gestantes [dissertação]. Recife: Univ. de Pernambuco; 2000.

21.Varner M. General medical and surgical diseases in pregnancy. In: Scott JR, Disaia PJ, Hammond CB, Spellacy WN, editors. Danforth's Obstetrics and Gynecology. $6^{\text {th }}$ ed. Philadelphia: J. B. Lippincott; 1990. p.495-533.

22.Sood A, Singh D, Mehta S, Midha V, Kumar R. Response to hepatitis $B$ vaccine in preterm babies. Indian J Gastroenterol 2002; 21:52-4.

23.Kirmani KI, Lofthus G, Pichichero ME, Voloshen T, D'Angio CT. Seven-year follow-up of vaccine response in extremely premature infants. Pediatrics 2002;109:498-504.

24.Patel DM, Butler J, Feldman S, Graves GR, Rhodes PG. Immunogenicity of hepatitis B vaccine in healthy very low birth weight infants. J Pediatr 1997; 131:641-3.

25.Lau YL, Tam AY, Ng KW, et al. Response of preterm infants to hepatitis B vaccine. J Pediatr 1992; 121:962-5. 